Pierre Talbot Patricia Jouvenne

\title{
Le potentiel neurotrope des coronavirus
}

Les coronavirus sont de grands virus à $A R N$ possédant un génome de plus de 30000 nucléotides et impliqués, chez l'homme, dans près du tiers des infections aiguës du tractus respiratoire. Du fait de leur complexité et de la bénignité des symptômes provoqués, ils restent mal connus et sont rarement recherchés dans des maladies humaines. Il existe cependant un modèle murin d'atteinte neurologique par coronavirus avec induction d'une immunité contre la protéine basique de la myéline et démyélinisation rappelant les signes de la sclérose en plaques. De plus, un petit fragment de cinq acides aminés d'une protéine coronavirale humaine est identique à un motif de la protéine basique de la myéline humaine, situé à proximité de zones reconnues par les lymphocytes de malades atteints de sclérose en plaques. Les coronavirus font enfin partie des virus repérés (du fait de la présence de particules virales ou d'anticorps) dans cette affection. Tous ces indices devraient stimuler les recherches sur cette espèce de virus et son rôle éventuel dans des maladies humaines graves avec démyélénisation, avant tout la sclérose en plaques.

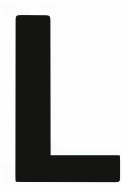

es coronavirus sont encore trop souvent absents de la littérature médicale, ce qui reflète indéniablement un manque de travaux et de connaissances sur ce sujet. D'abord isolés au milieu des années soixante chez des patients atteints de rhumes, il est maintenant reconnu que les coronavirus humains sont responsables de 15 à $35 \%$ des infections aiguës du tractus respiratoire, les symptômes cliniques pouvant d'ailleurs être reproduits expérimentalement chez des volontaires [1]. Les principaux autres agents infectieux associés à ce type de maladie sont les rhinovirus et les virus para-influenza et influenza. Dès l'enfance, la très grande majorité de la population présente une séroconversion face aux antigènes coronaviraux, ce qui souligne la haute prévalence des infections causées par ces virus apparemment endémiques $[1,2]$. Les maladies qu'on leur attribue actuellement, quoique bénignes, entraînent néanmoins des pertes économiques notables. En outre, l'importance médicale des coronavirus pourrait être plus grande que ce que nos connaissances encore superficielles sur cette famille

P. Talbot. 


\section{RÉFÉRENCES}

1. McIntosh K. Coronaviruses. In : Field BN, Knipe DM, et al., eds. Virology, $2^{\text {nd }}$ ed. New York : Raven Press, 1990 857-64.

2. Chambon M, Laveran H, L'Haridon R, Peigue-Lafeuille $\mathrm{H}$, Trimolet $\mathrm{M}$, Beytout D. Enquête sérologique par immunofluorescence indirecte sur les infections à coronavirus $\mathrm{OC}_{43}$. Ann Inst Pasteur Virol 1987; 138 : 263-8.

3. Riski N, Hovi T. Coronavirus infections of man associated with diseases other than the common cold. J Med Virol 1980; 6 : 259-65.

4. Resta S, Luby JP, Rosenfeld CR, Siegel JD. Isolation and propagation of a human enteric coronavirus. Science 1985 ; 229 : 978-81.

5. Battaglia M, Passarani N, DiMatteo A, Gerna G. Human enteric coronaviruses further characterization and immunoblotting of viral proteins. J Infect Dis $1987 ; 155$ 140-3.

6. Tanaka R, Iwasaki Y, Koprowski HJ. Intracisternal virus-like particles in the brain of a multiple sclerosis patient. $J$ Neurol Sc 1976 ; 28 : 121-6.

7. Burks JS, DeVald BL, Jankovsky LD, Gerdes JC. Two coronaviruses isolated from central nervous system tissue of two multiple sclerosis patients. Science $1980 ; 209$ 933-4.

8. Salmi A, Ziola B, Hovi T, Reunanen M. Antibodies to coronaviruses $\mathrm{OC}_{43}$ and $229 \mathrm{E}$ in multiple sclerosis patients. Neurology 1982 ; 32 : 292-5.

9. Fishman PS, Gass JS, Swoveland PT, Lavi E, Highkin MK, Weiss SR. Infection of the basal ganglia by a murine coronavirus. Science 1985 ; 229 : 877-9.

10. Chaloner-Larson G, JohnsonLussenburg CM. Establishment and maintenance of a persistent infection of L132 cells by human coronavirus strain 229E. Arch Virol 1981; 69 : 117-29.

11. Collins AR, Sorensen O. Regulation of viral persistence in human glioblastoma and rhabdomyosarcoma cells infected with coronavirus $\mathrm{OC}_{43}$. Microbiol Pathol 1986 ; 1 : 573-82.

12. Spaan W, Cavanagh D, Horzinek MC. Coronaviruses. In : Van Regenmortel MHV, Neurath AR, eds. Immunochemistry of Virus. II. The Basis for Serodiagnosis and Vaccines. Amsterdam : Elsevier, 1990 : 359-79.

13. Holmes KV. Coronaviridae and their replication. In : Fields $\mathrm{BN}$, Knipe DM, et al, eds. Virology, $2^{\text {nd }}$ ed. New York : Raven Press, 1990 : 841-56.

14. Lai MMC. Coronavirus : organization, replication and expression of genome. $A n n$ Rev Biochem 1990; 44 : 303-33.

Tableau I

\section{IMPORTANCE MÉDICALE DES CORONAVIRUS}

\section{Maladies coronavirales reconnues}

- Infections respiratoires du type rhume (15 à $35 \%$ de ces infections)

\section{Maladies dans lesquelles les coronavirus ont été occasionnellement impliqués}

- Certaines pneumonies, périmyocardites, méningites et radiculites

- Infections gastro-intestinales : diarrhées, entérocolites nécrotiques

- Sclérose en plaques

- Maladie de Parkinson

de virus et l'absence d'outils diagnostiques appropriés nous permettent d'évaluer actuellement (Tableau I). Une étude séro-épidémiologique suggère, par exemple, leur implication dans l'étiologie de maladies plus graves telles que certaines pneumonies et périmyocardites, et peut-être des méningites et des radiculites [3]. D'autres observations faisant état de coronavirus entériques associés à certaines gastro-entérites graves, surtout chez les nouveau-nés et les enfants âgés de moins d'un an, devront être confirmées [1, 4, 5]. Enfin, les coronavirus figurent sur la longue liste des responsables potentiels de la sclérose en plaques (SEP), une maladie démyélinisante auto-immune qui se développe chez les personnes génétiquement prédisposées, à la suite de l'exposition à un facteur de l'environnement encore inconnu. L'implication neurologique des coronavirus reste largement à démontrer. Des particules coronaviriformes ont cependant été observées dans des coupes de cerveaux [6] et des coronavirus isolés [7] chez des patients atteints de SEP. De plus, des anticorps anticoronavirus ont été détectés fréquemment, et à des titres élevés, dans le liquide céphalo-rachidien de malades atteints de SEP [8]. Par ailleurs, la localisation histologique d'antigènes coronaviraux dans le cerveau de souris infectées rappelle l'histopathologie du syndrome post-encéphalitique parkinsonien ou de la maladie de Parkinson [9]. Il est à noter, par ailleurs, que les coronavirus humains partagent avec une multitude d'autres virus la propriété d'être à l'origine d'infections persistantes $[10,11]$, ce qui suggère la possibilité d'un processus pathogène à développement lent. Omniprésents, les coronavirus constituent une famille d'agents pathogènes apparentés sur les plans structural et fonctionnel. Leur implication dans des maladies plus ou moins débilitantes des voies respiratoires, du tractus gastro-intestinal ou du système nerveux central chez plusieurs mammifères et volatiles [12, 13], devrait donc nous apporter des indices précieux pour l'étude de la pathogénie coronavirale chez les êtres humains.

\section{Structure et réplication}

Morphologie. Les coronavirus forment une famille de virus enveloppés, dont les projections de surface, ou péplomères, espacées et en forme de bulbes, donnent une apparence caractéristique de couronne ceignant les particules virales (figure $1 A$ ), d'où leur nom proposé en 1968. Certains coronavirus, dont l'un des deux sérotypes humains connus, possèdent une seconde frange de péplomères plus petits qui sont associés à une activité hémagglutinante, facilitant la détection des virus, mais dont la fonction biologique demeure inconnue. La majeure partie de nos connaissances sur la structure moléculaire, la biologie et la pathogenèse des coronavirus provient des travaux réalisés sur les coronavirus murins [1, 12-14].

Génome et protéines. Avec plus de 30000 nucléotides, le génome viral représente le plus long ARN connu. Son mode particulier de réplication 


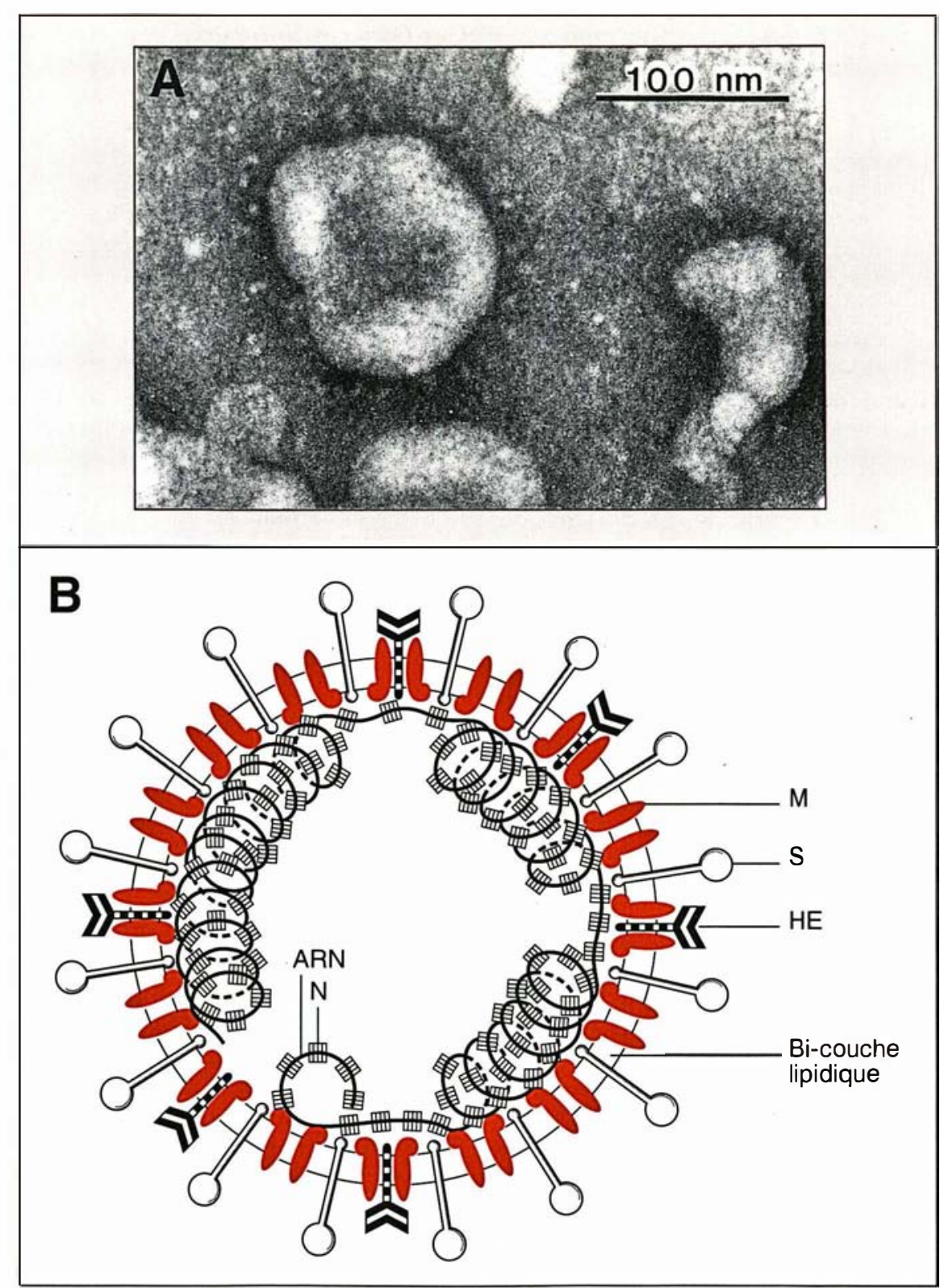

Figure 1. Structure du coronavirus. (A) Le coronavirus humain, souche prototype 229E, observé au microscope électronique après coloration négative (résultats non publiés). (B) Modèle structural des coronavirus : la protéine HE n'est présente que sur l'un des deux sérotypes humains du coronavirus, représenté par la souche prototype OC43. (D'après [13].)
[14] engendre une fréquence élevée de recombinaison. La séquence nucléotidique du génome d'un coronavirus murin suggère, par exemple, que l'hémagglutinine retrouvée chez certains coronavirus aurait été acquise lors d'une coinfection avec une souche du virus influenza [13]. Une recombinaison entre gènes coronaviraux et cellulaires serait, par ailleurs, concevable. Enfin, la réplication d'une si longue molécule d'ARN donne lieu à des mutations qui contribuent aussi à la variabilité génétique. Tout cela laisse entrevoir la capacité d'adaptation des coronavirus et inquiète quant à la possibilité d'apparition de variants dont le pouvoir pathogène pourrait être augmenté ou modifié à l'avantage du virus.

Trois ou quatre protéines structurales composent les virions (figure $1 B$ ). D'une part, la protéine $\mathrm{N}$ qui sert à encapsider l'ARN génomique et, d'autre part, deux ou trois glycoprotéines qui sont insérées dans l'enveloppe virale ravie à la cellule infectée lors de la morphogenèse des virions. La glycoprotéine de membrane $\mathbf{M}$ semble jouer un rôle essentiellement structural. La glycoprotéine de surface $\mathrm{S}$ forme les péplomères viraux. Elle est donc impliquée dans l'attachement des particules virales aux récepteurs présents à la surface des cellules susceptibles, l'antigène carcinoembryonnaire semblant constituer une cible privilégiée [15]. La protéine $\mathrm{S}$ joue aussi un rôle primordial dans la propagation virale par fusion cellulaire, suite à son insertion dans la membrane cytoplasmique des cellules infectées. Chez plusieurs coronavirus, cette activité de fusion est induite par le clivage protéolytique de la molécule. Ce clivage se produit de façon différente selon le type de cellule impliquée, ce qui pourrait influencer la pathogenèse comme dans le cas des virus de la rougeole et de l'immunodéficience acquise chez l'humain. Certains coronavirus, dont un des deux sérotypes humains, possèdent en plus une troisième glycoprotéine, nommée $\mathrm{HE}$ pour ses activités hémagglutinante et d'estérase. La fonction biologique de cette protéine demeure inconnue. Enfin, à l'exception d'une ARN polymérase ARN-dépendante, on ignore 


\section{RÉFÉRENCES}

15. Williams RK, Jiang GS, Holmes KV. Receptor for mouse hepatitis virus is a member of the carcinocmbryonic antigen family of glycoprotcins. Proc Natl Acad Sci USA 1991 ; 88 : 5533-6.

16. Callow KA. Effect of specific humora immunity and some non-specilic factors on resistance of voluntecrs to respiratory coronavirus infection. I Hyg Camb 1985 ; 95 : 173-89.

17. Holmes MJ, Callow KA, Childs RA, Tyrrell DAJ. Antibody dependent cellular cytotoxicity against coronavirus 229E infected cells. Br J Exp Pathol 1986; 67 581-6.

18. Lamontagne L, Descôtcaux JP, Jolicocur P. Mousc hepatitis virus 3 replication in $\mathrm{T}$ and $\mathrm{B}$ lymphocytes correlate with viral pathogenicity. J Immunol $1989 ; 142$. 4458-65.

19. Knobler RL, Lampert PW, Oldstone MBA. Virus persistence and recurring demyclination produced by a temperature sensitive mutant of MHV-4. Nature 1982 298 : $279-80$

20. Watanabe $\mathrm{R}$, Wege $\mathrm{H}$, ter Meulen V Adoptive transfer of EAE-like lesions from rats with coronavirus-induced demyclinating encephalomyclitis. Nature 1983 ; 305 : 150-3.

21. Wang FI, Stohlman SA, Fleming JO Demyclination induced by murine hepatitis virus JHM strtain (MHV-4) is immuno logically mediated. J Neuroimmunol 1990 ; $30: 31-41$.

22. Massa PT, Dörries R, ter Meulen V. Viral particles induce la antigen expression on astrocytes. Nature 1986 ; 320 : 543-6.

23. Suzumura A, Lavi E, Wciss SR, Silberberg $\mathrm{DH}$. Coronavirus infection induces $\mathrm{H}-2$ antigen expression on oligodendrocytes and astrocytcs. Science $1986 ; 232$ : 991-3.

24. Williamson JSP, Stohlman SA. Effective clearance of mouse hepatitis virus from the central nervous system requires both CD4 + and CD8 + T-cells. J Virol 1990 ; $64: 4589-92$

25. Lamarre A, Lecomte J, Talbot PJ Anti-idiotypic vaccination against murinc coronavirus infection. J Immunol $1992 ; 147$ 4256-62.

26. Danicl C, Talbot PJ. Protection from lethal coronavirus infection by affinitypurified spike glycoprotein of murine hepatitis virus, strain A59. Virology $1990 ; 174$ : 87-94

27. Talbot PJ, Dionne G, Lacroix M. Vaccination against lethal coronavirus-induced encephalitis with a synthetic decapcptide homologous to a domain in the predicted la fonction des quatre ou cinq protéines non structurales du coronavirus

Infection. Le degré de cytopathie induit par une infection coronavirale varie selon la souche virale et la cellule cible. Il va de la formation de cellules géantes multinucléćes (syncytia), suivie de la lyse cellulaire, à l'absence quasi complète d'effet cytopathique accompagnant une production continue ou cyclique de virus infecticux. Ces dernières infections persistantes donnent lieu à la production de mutants dont la virulence est modifiće. Le rôle de ces mutants ou de particules défectueuses dans la persistance n'est pas encore compris [13]. Le bourgeonnement intracellulaire des coronavirus constitue un avantage certain car il permet la propagation de l'infection à l'abri du système immunitaire.

\section{Pathogenèse et réponse immunitaire}

Coronavirus humains. La pathogenèse de l'infection coronavirale chez l'humain ainsi que les mécanismes de défense immunitaire contre celle-ci sont peu connus. La destruction localisće des cellules épithéliales ciliées, à la suite de leur infection par le coronavirus, contribue fort probablement au développement des maladies respiratoires et peut-être gastro-intestinales [1]. L'inoculation de volontaires a permis de montrer l'importance des anticorps circulants et locaux, ces derniers étant du type IgA, dans l'atténuation des symptômes et l'élimination du virus [16]. Il semble que les anticorps neutralisants soient dirigćs contre les péplomères viraux [1]. La destruction immunitaire des cellules infectćes pourrait impliquer un mécanisme de cytotoxicité cellulaire dépendante des anticorps [17]. Malheureusement, l'infection coronavirale ne produit qu'une immunité spécifique de la souche virale impliquée et l'hétérogénćité antigénique, même à l'intćrieur d'un seul sérotype, favorise plusicurs infections consćcutives [1]. L'administration nasale d'interfćron $\alpha$ semble, en revanche, davantage prometteuse pour réduire la durée et la sévéritć des rhumes coronaviraux, que pour atténuer les infections rhinovirales [1].
Coronavirus animaux. Les modèles animaux des infections coronavirales nous fournissent des informations précieuses sur la biologie de ces virus. Le coronavirus de rat produit une rhinotrachéite accompagnée d'une légère pneumonie interstitielle localisée et d'une infiltration cellulaire. Le virus de la bronchite infectieuse aviaire cause également une maladie respiratoire, mais l'infection se propage rapidement au système reproducteur et aux reins du poulet. Par ailleurs, une souche coronavirale murine induit une immunodépression chez l'animal, associée à la réplication du virus dans les lymphocytes B et $T$ [18]. Toutefois, le modèle animal qui laisse le plus songeur quant à l'importance médicale de ces virus est celui de l'infection coronavirale du système nerveux central de la souris et du rat. Des souches ncurotropes du coronavirus murin provoquent en effet des maladies neurologiques qui présentent des caractéristiques pathologiques similaires à celles rencontrćes dans des maladies humaines telles que la SEP. Ces modèles expérimentaux permettent la dissection des déterminants moléculaires de la maladie et l'analyse des interactions entre le virus et le système immunitaire.

Coronavirus neurotropes. Des souris adultes infectées par un coronavirus murin neurotrope développent habituellement une encéphalomyćlite accompagnée d'une infection des cellules gliales et neuronales. Il y a alors plus de $95 \%$ de mortalité. Les quelques animaux qui survivent à cette maladie aiguë présentent une affection de la matière blanche caractérisée par l'apparition des plaques de démyélinisation dans le cerveau et la moelle épinière, suivie d'une remyćlinisation puis d'une démyélinisation récurrente. Deux processus pathologiques différents pourraient expliquer le développement de ce type de maladie démyélinisante. Il pourrait s'agir, d'une part, de l'infection et de la destruction des oligodendrocytes, les cellules responsables de la synthèse de la gaine de myéline (les antigènes viraux sont d'ailleurs détectés dans ces cellules durant au moins une année) [19]. Il pourrait, d'autre part, s'agir d'une attaque auto-immunitaire du système nerveux central résultant 


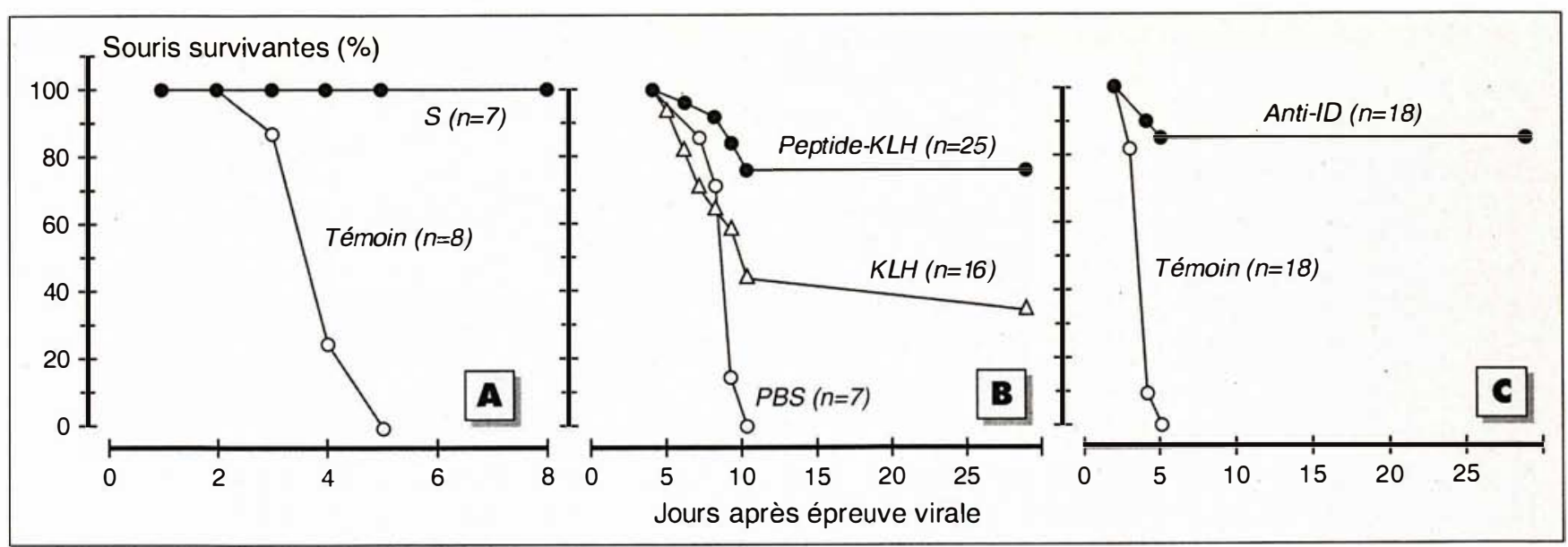

Figure 2. Vaccination contre l'encéphalite coronavirale. (A) Souris vaccinées avec la protéine virale de surface $S$ purifiée par chromatographie d'affinité. (D'après [26].) (B) Souris vaccinées avec un peptide synthétique homologue à une région de dix acides aminés de la protéine de surface $S$ du coronavirus murin neurotrope. Le peptide est couplé à une molécule porteuse, I'hémocyanine de patelle (KLH) pour le rendre immunogène. Les groupes contrôles sont immunisés avec du tampon (PBS) ou la molécule porteuse. La protection partielle, statistiquement non significative, conférée par celle-ci pourrait être expliquée par une homologie fortuite observée entre cette protéine et une molécule virale. (D'après [27].) (C) Souris vaccinées avec des anticorps anti-idiotypiques produits contre un anticorps monoclonal reconnaissant la protéine de surface $S$. Le groupe témoin est immunisé avec des immunoglobulines normales. (D'après [25].)

de l'infection virale. Cette dernière hypothèse est renforcée par l'observation d'une sensibilisation à la protéine basique de la myéline (PBM) des lymphocytes des animaux infectés que révèle l'apparition d'une encéphalomyélite allergique expérimentale après le transfert adoptif de ces cellules à des animaux non infectés [20]. Cette maladie auto-immune peut être reproduite expérimentalement par l'injection de PBM. De plus, un traitement immunosuppresseur prévient le développement de la démyélinisation viro-induite chez la souris [21]. Enfin, il a été démontré récemment que des particules coronavirales peuvent induire l'expression d'antigènes d'histocompatibilité de classes I et II sur des cellules gliales du système nerveux central, ce qui suggère l'activation, à ce niveau, de réponses immunitaires à médiation cellulaire [22, 23].

Chez l'animal, divers types de mutants viraux induisent un processus pathologique modifié où les oligodendrocytes sont infectés et les neurones épargnés. Cette maladie, caractérisée par une démyélinisation chronique et récurrente plutôt que par une encéphalite létale, est aussi $m / s n^{\circ} 2$, vol. 8, février 92 observée après le transfert passif de certains anticorps monoclonaux ou de lymphocytes $\mathrm{CD}^{+}{ }^{+}$. Il semble que l'élimination complète de l'infection virale nécessite des lymphocytes $\mathrm{CD}^{+}{ }^{+}$et $\mathrm{CD}^{+}{ }^{+}[24]$. Par ailleurs, chez la souris, des facteurs génétiques déterminent la susceptibilité à la maladie coronavirale, et plusieurs gènes récessifs autosomaux contrôlant la résistance ont été identifiés [13].

Déterminants moléculaires. L'importance de la glycoprotéine S dans la virulence, ainsi que dans le tropisme et l'induction d'une réponse immunitaire protectrice, ne fait maintenant plus de doute. Des anticorps monoclonaux neutralisants spécifiques de certains sites antigéniques de la protéine $\mathrm{S}$ protègent passivement contre l'encéphalite coronavirale. Il est également possible de préparer un vaccin efficace de type antiidiotypique constitué d'anticorps produits contre un anticorps monoclonal protecteur [25] (figure 2C). Un vaccin sous-unitaire constitué de la protéine $S$ purifiée [26] et un vaccin synthétique composé d'un décapeptide couplé à une molécule porteuse [27] peuvent également protéger les souris contre la maladie (figures $2 A$ et $2 B$ ). Par ailleurs, une région essentielle en tant que cible de l'immunité protectrice, et dont la conservation sur plusieurs souches virales suggère l'importance biologique, a été localisée précisément sur la protéine S [12]. Enfin, des mutants viraux beaucoup moins virulents et de tropisme altéré, résultant apparemment de modifications de cette protéine clé de la biologie coronavirale, ont pu être isolés sous la pression sélective de certains anticorps monoclonaux neutralisants et spécifiques à des sites antigéniques précis sur la protéine $\mathrm{S}[12,28]$.

D'autres protéines virales jouent fort probablement un rôle important dans les interactions virus-hôte. Certains anticorps monoclonaux dirigés contre la glycoprotéine $M$ peuvent, par exemple, neutraliser l'infection virale in vitro en présence de complément $[12,13]$, et même protéger contre l'encéphalite in vivo [29]. La protéine $\mathrm{N}$ peut également représenter la cible d'anticorps protecteurs [12], et il est concevable, par analogie avec d'autres infections virales, qu'elle participe à l'induction d'une réponse immunitaire à médiation cellulaire. 


\section{RÉFÉRENCES}

28. Parker SE, Gallagher TM, Buchmeïer MJ. Sequence analysis reveals extensive polymorphism and evidence of deletions within the E2 glycoprotein gene of several strains of murine hepatitis virus. Virology $1989 ; 173$ : 664-73.

29. Fleming JO, Shubin RA, Sussman MA, Casteel N, Stohlman SA. Monoclona antibodies to the matrix (E1) glycoprotein of mouse hepatitis virus protect mice from encephalitis. Virology 1989 ; 168 : 162-7.

30. Fritz RB, McFarlin DE. Encephalitogenic epitopes of myelin basic protein. In Sercarz E, eds. Antigenic Determinants and Immune Regulation Basel: Karger, 1989 : 101-25.

31. Ota K, Matsui M, Milford EL, Mac kin GA, Weiner HL, Hafler DA. T-cell recognition of an immunodominant myelin basic protein epitope in multiple sclerosis Nature 1990 ; 346 : 183-7.

32. Oldstone MBA. Molecular mimicry and autoimmune disease. Cell $1987: 50: 819-20$

33. Jouvenne $P$, Mounir S, Stewart JN Richardson CD, Talbot PJ. Sequence analy sis of mRNAs 4 and 5 of human coronavirus $229 \mathrm{E}$ : evidence for polymorphism and homology with myelin basic protein. Virus Res 1992 (sous presse).

34. Hashim G, Vandenbark AA, Gold DP, Diamanduros $T$, Offner $H$. T-cell lines specific for an immunodominant epitope of human basic protein define an encephalitogenic determinant for experimental autoimmune encephalomyelitis-resistant LOU/M rats. J Immunol $1991 ; 146$ : 515-20.

35. Richert JR, Robinson ED, Deibler GE Martenson RE, Dragovic LJ, Kies MW. Human cytotoxic T-cell recognition of a synthetic peptide of myelin basic protein Ann Neurol $1989 ; 26: 342-6$.

36. Jingwu $\mathrm{Z}$, Chou $\mathrm{CH}$, Hashim $\mathrm{G}$ Medear R, Raus JCM. Preferential peptide specificity and HLA restriction of myelin basic protein-specific $\mathrm{T}$-cell clones derived from MS patients. Cell Immunol $1990 ; 129$ : 189-98.

37. Pearson J, Mims CA. Differential susceptibility of cultured neural cells to the human coronavirus $\mathrm{OC}_{43}$. J Virol 1985 ; 53 : 1016-9.

38. Myint S, Harmsen D, Raabe T, Siddell SG. Characterization of a nucleic acid probe for the diagnosis of human coronavirus-229E infections. J Med Virol 1990 ; $31: 165-72$.

39. Stewart JN, Mounir S, Talbot PJ. Detection of coronaviruses by the polymerase chain reaction. In : Becker Y, Darai G, eds. Diagnosis of Human Viruses by Polymerase Chain Reaction (PCR) Technology; Frontiers of Virology. Heidelberg : Springer-Verlag, 1992

\section{Voies de recherche fournies par les coronavirus animaux}

Maladie neurologique. La protéine basique de la myéline et la protéine protéolipidique constituent les protéines les plus abondantes dans le système nerveux central. Chez plusieurs espèces animales, l'injection de ces protéines provoque une encéphalomyélite allergique expérimentale, une maladie neurologique auto-immune, impliquant des lymphocytes $\mathrm{T}$ spécifiques au tissu cible, et dont les caractéristiques cliniques et histopathologiques ressemblent beaucoup à la SEP, maladie démyélinisante la plus répandue chez l'homme. La séquence primaire de la PBM est hautement conservée chez plusieurs espèces animales et quelques-uns de ses déterminants encéphalitogènes, qui varient selon l'espèce inoculée, ont été identifiés $[30,34]$. Bien que les sites encéphalitogènes chez l'être humain ne puissent être directement caractérisés, l'étude de la spécificité moléculaire des lymphocytes isolés chez des patients permet de les identifier. Une région immunodominante pour la reconnaissance de la PBM par les lymphocytes $\mathrm{T}$ de patients atteints de SEP est maintenant connue [31]. Un des mécanismes d'induction possibles d'une maladie auto-immune d'origine virale consiste en un partage de séquences protéiques entre le virus et l'organisme hôte, un phénomène relativement fréquent [32]. Ce mimétisme moléculaire peut être fortuit. Nous avons remarqué que des antisérums spécifiques au coronavirus murin reconnaissent l'hémocyanine de patelle, une protéine extraite d'un mollusque que l'on utilise comme molécule porteuse pour rendre immunogènes des peptides synthétiques. Cela pourrait expliquer la protection partielle contre l'encéphalite coronavirale qui est conférée par cette molécule (figure 2B). En revanche, le mimétisme moléculaire pourrait aussi être acquis par le virus au cours de l'évolution afin de contribuer à son potentiel pathogène. Ainsi, lorsque la réponse immunitaire vise des régions partagées entre des protéines virales et cellulaires, l'auto-immunité induite peut avoir des conséquences pathologiques malheureuses, comme c'est le cas lorsque le site encéphalitogène de la PBM constitue la cible de cette attaque immunitaire. Nous avons remarqué une homologie de cinq acides aminés (taille minimale des épitopes reconnus par les lymphocytes $T$ et les anticorps) entre une protéine non structurale d'une souche coronavirale humaine et la PBM humaine (figure 3) [33]. Un calcul mathématique indique que les chances que cette homologie soit issue d'un pur hasard sont de un sur plus de trois millions. Cette région conservée est associée à des sites encéphalitogènes de la PBM identifiés après l'injection des peptides correspondants à des animaux de laboratoire [30, 34]. Elle se trouve à quelques résidus en aval de la région reconnue préférentiellement par le système immunitaire de patients atteints de SEP [31], et est la cible de lymphocytes retrouvés chez certains de ces patients [35, 36]. Enfin, on peut signaler que la susceptibilité des cellules nerveuses à l'infection coronavirale in vitro a déjà été rapportée pour un sérotype [37]. Bien que l'importance médicale des coronavirus reste à établir, de telles observations montrent le bien-fondé de l'étude du neurotropisme de ces virus.

\section{Diagnostic}

Les laboratoires de diagnostic n'incluent pas les coronavirus dans leur panoplie de tests. Rétrospectivement, quelques études épidémiologiques sur la prévalence des infections respiratoires ont été réalisées à l'aide d'un test immunoenzymatique du type ELISA. Seuls, cependant, les anticorps dirigés contre les deux sérotypes connus sont détectables, et la présence d'autres sérotypes demeure une possibilité bien réelle [1]. L'isolement direct de coronavirus après l'inoculation de cellules en culture, quoique très difficile étant donné leur grande spécificité tissulaire et d'espèce, a fait également l'objet de quelques travaux. Actuellement, le diagnostic des infections entériques mettant en cause des coronavirus se limite à l'observation de particules caractéristiques au microscope électronique, ce qui rend les résultats incertains. Enfin, seuls quel- 


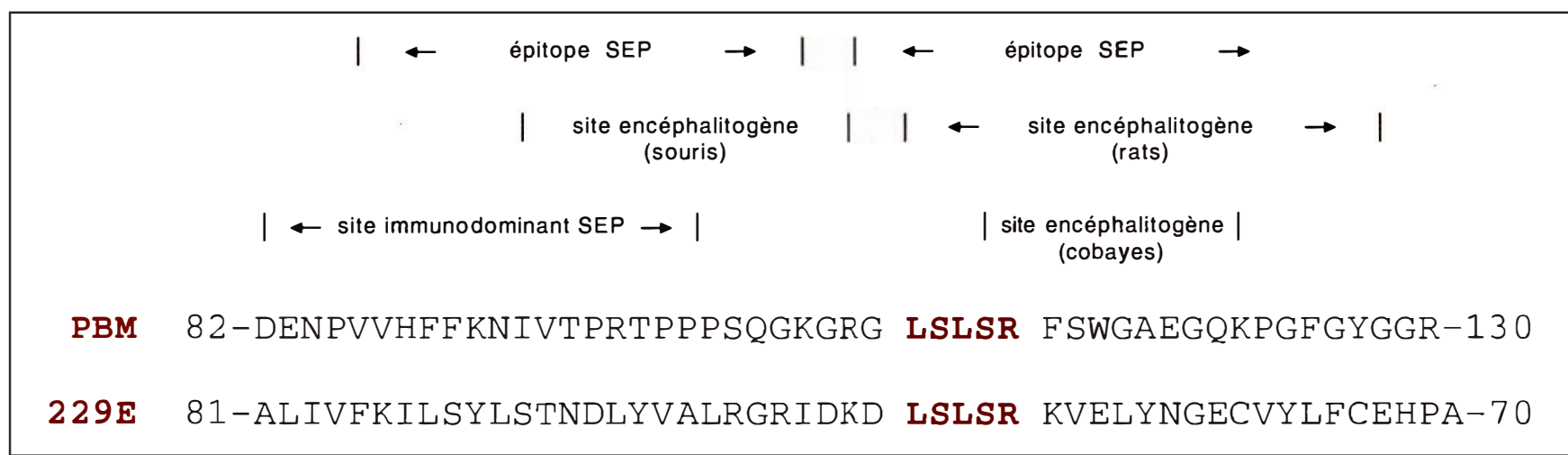

Figure 3. Homologie entre une protéine virale et la protéine majeure de la myéline. Une séquence de cinq acides aminés d'une protéine non structurale de la souche prototype $229 E$ du coronavirus humain se retrouve sur la protéine basique de la myéline (PBM), près d'un site immunodominant reconnu par des lymphocytes de personnes atteintes de sclérose en plaques (SEP) [31], en aval ou dans le cadre de deux régions vues par des lymphocytes de certains de ces patients [35, 36], et associée à des sites encéphalitogènes pour la souris [30], le rat [34] et le cobaye [30]. (D'après [33].)

ques travaux isolés se sont concentrés sur l'implication neurologique des coronavirus, apportant des résultats encore très controversés. Heureusement, des efforts commencent à être faits pour développer des méthodes de diagnostic rapides et sensibles par hybridation moléculaire [38] et amplification par polymérisation en chaîne (PCR) [39]. Ces développements permettront d'accélérer la recherche sur l'épidémiologie et la pathogenèse des coronavirus humains.

\section{Conclusion}

A l'heure actuelle, l'importance médicale reconnue des coronavirus se limite à des infections respiratoires bénignes qui, bien que très répandues, n'ont pas encore mérité l'attention des autorités médicales. Malgré quelques indices touchant leur implication dans des inf ections gastro-intestinales et neurologiques, les efforts de recherche sur les coronavirus entériques et neurotropes demeurent bien faibles. A peine avons-nous levé le voile sur l'importance médicale des coronavirus qu'apparaît la nécessité de développer de nouveaux outils moléculaires qui permettront de vérifier l'implication médicale soupçonnée de ces virus insidieux, puis d'envisager la prévention et le traitement. Nous nous devons d'explorer les voies de recherche que nous fournissent les coronavirus animaux, afin de vérifier l'existence du neurotropisme coronaviral chez l'être humain

\section{Summary}

Neurotropic potential of coronaviruses

Coronaviruses are ubiquitous pathogens responsible for up to a third of common colds. Moreover, circumstantial evidence has linked them to some diarrheas and neurological disorders such as multiple sclerosis. This family of enveloped RNA viruses has so far received little attention from the medical community, a reflection of the paucity of knowledge on their biology and the lack of widely used diagnostic tests. Fortunately, animal models of coronavirus infections have started to reveal important information on viral pathogenesis and interaction with the immune system. For example, acute encephalitis caused by neurotropic murine coronaviruses can be prevented by vaccination with a purified viral surface protein or a synthetic peptide homologous to an important determinant of this molecule. Prevention is also possible by passive transfer of monoclonal antibodies against this protein, by active immunization with anti-idiotypic antibodies prepared against a monoclonal antibody, or by the use of attenuated viral mutants that have lost these pathogenesis determinants. Interestingly, animals infected with this virus are immunologically sensitized to myelin basic protein, a major component of the myelin sheath surrounding nerve fibers. Immunosuppressed animals do not develop a demyelinating disease. Coronavirus-induced neurological disease may therefore be immune-mediated. A recently observed sequence homology between a human coronavirus protein and a region of myelin basic protein situated near an immunodominant site recognized by lymphocytes of multiple sclerosis patients may provide a link between the animal model and the possible neurological involvement of human coronaviruses. State-ofthe-art diagnostic technologies such as nucleic acid hybridization and polymerase chain reaction are starting to be applied to coronaviruses and should provide important information on the suspected medical importance of these insidious viruses. Knowledge gained from animal models could then serve as a basis for the development of preventive and curative measures. 